\title{
A Solution to the Hyper Complex, Cross Domain Reality of Artificial Intelligence: The Hierarchy of AI
}

\author{
Dr Andrew Kear ${ }^{1}$ \\ Faculty of Media and Communication \\ Bournemouth University \\ England
}

\author{
Sasha L. Folkes ${ }^{2}$ \\ Marketing Intelligence \\ London
}

\begin{abstract}
Artificial Intelligence (AI) is an umbrella term used to describe machine-based forms of learning. This can encapsulate anything from Siri, Apple's smartphone-based assistant, to Tesla's autonomous vehicles (self-driving cars). At present, there are no set criteria to classify AI. The implications of which include public uncertainty, corporate scepticism, diminished confidence, insufficient funding and limited progress. Current substantial challenges exist with AI such as the use of combinationally large search space, prediction errors against ground truth values, the use of quantum error correction strategies. These are discussed in addition to fundamental data issues across collection, sample error and quality. The concept of cross realms and domains used to inform AI, is considered. Furthermore there is the issue of the confusing range of current AI labels. This paper aims to provide a more consistent form of classification, to be used by institutions and organisations alike, as they endeavour to make AI part of their practice. In turn, this seeks to promote transparency and increase trust. This has been done through primary research, including a panel of data scientists / experts in the field, and through a literature review on existing research. The authors propose a model solution in that of the Hierarchy of AI.
\end{abstract}

Keywords-Artificial intelligence; classification; ground truth value; Hierarchy of AI; Model of AI

\section{INTRODUCTION}

A great deal of public funded investment is going into AI and yet the authors propose that there are still some fundamental issues with the different classifications and thus understanding of AI. These in turn lead to under confidence which could be overcome with more logical classification. The literature on AI is vast and multifaceted. Below, the authors have grouped together a series of definitions that vary according to the source:

AI can be defined as "any system . . . that generates adaptive behaviour to meet goals in a range of environments can be said to be intelligent" [23];

AI can be seen as "intelligent systems" that "are expected to work, and work well in many different environments [27]. Their property of intelligence allows them to maximize the probability of success even if full knowledge of the situation is not available',

AI is also defined as a division of computer science, in particular, "the study of the relation between computation and cognition', [5];
Others [61] note how AI is a "big field" that can be defined as "the study of agents that receive precepts from the environment and perform actions".

In addition to the above there are sources that argue $\mathrm{AI}$ is the wrong term entirely:

Psychometric Artificial Intelligence (PAI) is according to [9] more suitable, since it refers to "building informationprocessing entities capable of at least solid performance on all established, validated tests of intelligence and mental ability", (including artistic and literary creativity/ mechanical ability);

An alternative view [70] argues that the lack of consistency in definitions goes beyond semantic differences, as it poses a threat to developments in the field. With multiple definitions, "progress made under one characterization of AI is not viewed as success by others who operate under a different perception of it" [70]. This results in diminished confidence in the field, as well as "promote(ing) premature conclusions of what can and cannot be accomplished and limit progress and funding along research paths".

It is argued [45] that much of the trouble around defining AI is due to the issues with "intelligence". In their 2007 paper, 70 definitions of "intelligence", were gathered to display the disparity. An alternative view [2] highlights how, "curiously, the lack of a precise, universally accepted definition of AI probably has helped the field to grow, blossom, and advance"; several scholars argue for "changing the language used around AI to sharpen its conceptual clarity" [43].

This is particularly relevant, as awareness of AI is rising, albeit with muddled meaning. In a nationally representative survey by [14] which interviewed 1078 respondents, just 42\% were able to provide a credible definition of AI. A quarter of respondents described AI as relating to robots, and of all responses, the majority had the view that AI causes significant anxiety. This is problematic for industries such as eHealth (electronic health), as it is reliant on patient trust; similarly with schooling, public services and retail.

Despite several breakthroughs, AI is not without criticism. It is highlighted [79] that while accidents involving software or robotics "can be traced back to the early days of such technology", AI failures "are directly related to the mistakes produced by the intelligence such systems are designed to exhibit'. Instead of learning an intended function, an AI system can adapt an alternate function to reach the predefined 
goal. In 2016, OpenAI trained an AI agent to play CoastRunners, an online multiplayer game. Instead of finishing the course, the AI agent found a way of winning the game without completing the course: by repeatedly turning around in a circle timed to coincide with reappearing targets. Though somewhat harmless in this context, the above example highlights the dangers of reinforcement learning using imperfect proxies [79].

In more practical settings, AI systems have been known to stereotypically assign professions to genders on LinkedIn and fail to recognise ethnic differences in smartphone ID systems, in the case of Apple's iPhone X [11]. In extreme cases, AI failures have resulted in death: with Uber's self-driving car crashing into a pedestrian in 2018 as an example. More recently in the news, DeepFakes have seen celebrities faces imposed onto pornography, political speeches and more. Additionally, issues with AI determining which inmates get parole have also been highlighted and subsequently noted [72] where "its potential dangers are serious and far-reaching: if video evidence is no longer credible, this could further encourage the circulation of fake news". While the harmful effects of the above are indisputable, it raises the importance of training such systems on unbiased data sets, $\mathrm{x}$ and $\mathrm{y}$. In addition to RWD sets.

\section{DATA SCIENCE AND QUALITY}

There are issues with real world data (RWD) as often it is not generally collected for research purposes. Issues with the data includes non-rigorous data collection, non-purposive sample selection, episodic and / or incorrect timelines, containing data collection biases, reactive, and at best can only offer partial snapshots. As a result, RWD can also be generally messy and sparse, and requires statistically rigorous and valid methods to clean the data and employ error correct to overcome data inconsistencies. The process of careful data identification, prioritisation and inclusion, using both structured and unstructured data, can be critical for valid data analysis and subsequently real world evaluation (RWE). How issues of missing data are filled can often invalidate the findings and yet a system of independent data regulation and validation is not present. Thus the authors contend that transparency on the type and amount of error correction is essential to build more understanding and subsequently trust in AI systems. In the context of healthcare where often crucial information related to molecular biomarkers or end-points data can be missing, the missing data gaps may be filled by bridging to alternative data sources [48]. Data scientists are required to identify and adjust for confounding factors such as demographics, socioeconomics, psychographic and behavioural data. Further complications exist depending on the particular domain or even combination of domains in question. As such it is important for more explainable AI and research therein, to improve transparency of AI [41].

Genetic predispositions and / or Neurological processing may offer a baseline before conducting in-depth analyses. RWD is also subject to selection bias, as cohort selection and treatment decisions in clinical practice are not random. Essential to acquisition of relevant data assets, guidelines on design and validation of RWE studies can help in minimizing some sources of bias and inconsistencies [76]. In addition, standards for the development and maintenance of data assets needs to keep up with the rapid evolution of RWD. The use of legacy data systems may inhibit or prevent large scale predictive accuracy and yet be fundamentally important to the task at hand. The diversity and complexity of systems, data types, data locations and data availability means that there is often a lack of interoperability which heightens complexity for any data collaborations. At a micro level within organisations data is often held in different data systems. In such situations, there is undoubtedly a need to implement standardisation and maintain robust quality assurance (QA) quality control (QC) practices to support data robustness.

\section{Current State OF AI}

It is generally regarded that deep learning, a subset of machine learning, is at the frontier of artificial intelligence research. Deep learning consists of multi-layered synthetic networks that are modelled on the human brain: namely, neural networks [68]. These deep learning networks have a nature of interoperability that is fundamental to the increasing potential of AI. Ideas about deep learning are not new, however. In 1943, [47] first discussed the notion of "neurons as elementary adaptive nonlinear processing units" [68] as opposed to logic-based units. Before this, analogue computers were thought of as logic-based, but with [47] ideas, the realm of possibility opened up. In the following years, computers had not quite reached the ability to analyse vast sets of data, though contemporary developments in technology have made this possible today.

Deep Face, for example, is a nine-layered neural network with over 110 million parameters created by Facebook that can identify human faces in photographs. Similarly, Deep Net which has been trained on over 150 million images from Google Net can identify facial similarities with accuracy levels of 98.73 and $96.12 \%$ [57]. However the issue of error correction amount and type is not presented or publicly available.

\section{CAtegorisation OF AI}

The assertion that Artificial intelligence (AI) seeks to process, understand and respond to data in the same ways which humans would [54] starts to become hyper complex considering the above human intelligence with the aim of AI to be anthropomorphic in nature and whereby the algorithms allow AI systems to mimic human cognitive functions to solve problems [38]. The next section considers some artificial intelligence fundamentals and as such the 4 basic AI concepts of 1, Categorisation - where metrics are created relevant to the domain, then 2, Classification - where the data is analysed to determine the most relevant to solving the problem, followed by $\mathbf{3}$, Dimensions and types of intelligence, followed by $\mathbf{4}$, Machine / Deep Learning which on a basic level involves anomaly detection, clustering, deep learning and analysis. Finally 5, Collaborative filtering where patterns are detected across large data sets resulting in certain forecasts, predictions or entailments. A key consideration in any new classification of AI is that of interpretability and explainability, as is the key criteria suggested to establish cause and effect [41] in scientific theory and should be part of the AI decision making 
explanation [43] and advise "re-framing conversations around machine autonomy to foreground human actors and the broader sociotechnical context in which such systems are embedded".

This following section seeks to break down commonalities among categorisation of AI; with the base level of Narrow / Weak AI - which lacks the ability to understand context but can perform simple demonstrative tasks;

At the central level is General AI; where it is able to understand context and make inferences from it and also operates on little to no information, and exhibit powers of reasoning and creativity; finally Super AI - which possesses an intellectual capacity far superior to that of a human beings. [36]. An additional consideration can be given to the methodology of AI whereby the training of it can be considered and includes; Training AI - can learn and improve over time; and Inference AI - requires human interference to make more relevant suggestions such that [55] "expertise in epistemology, critical thinking and reasoning are crucial to ensure human oversight of the artificial intelligent judgements and decisions that are made, because only competent human insight into AI-inference processes will ensure accountability". This method of classifying AI shares some similarities, but is advanced, with the following where AI has been classified is by the level of human systems interaction and includes; Supervised AI - which requires human monitoring and feedback; Unsupervised AI - the unsupervised suffers from the lack of "expert" touch (in the context of dermatology) during the training [1] and could be considered Black Box AI which does not require human interference, and Reinforcement AI whereby occasional human interference is needed. The combination method or the [1] "semi-supervised learning" method has also been introduced, which utilizes a small amount of labelled data and a larger amount of unlabelled data.

Beyond the above and to confuse matters further, there are also ways of classifying AI according to its potential:

- Expert systems, Analytical AI, Human-inspired AI, Humanised AI

The determining factors of which are listed as follows [42]

- Cognitive Intelligence

- Emotional Intelligence

- Social Intelligence

- Artistic Creativity

Clearly there are a number of similarities across the various labels and categories. In addition there are some basic yet important considerations pertaining to the level of human involvement, the type and level of intelligence that will feed forward into the design of the proposed model. However there are some gaps in relation to new areas of, in addition to new streams of intelligences, and their overall contribution to AI.

\section{DIMENSIONS OF AI}

In order to address issues of classifications there is a need to further review AI. Central to any discussion of AI should be human intelligence, Abstract Reasoning Corpus, Skill acquisition efficiency etc. However the current performance of AI should be considered as becoming far greater than human intelligence. A great deal is written about the abilities of AI systems to outperform humans in games, calculations and other narrow fields but, this is where key issues arise due to the differences in how humans might process the data in different domains. One of the key components of intelligence is cognitive learning which involves the acquisition of knowledge and internal mental structures through cognitive processes such as thinking, problem solving, language and information processing [69].

An interesting assertion [15] argues that intelligence cannot be measured by skill at a particular task or set of tasks. When we consider Abstraction and Reasoning Corpus (ARC) which is said to measure general fluid human intelligence, then a number of key elements need to be considered as important. These include that of skill acquisition efficiency which subsequently highlights concepts of scope, generalised difficulty, priors and experience [15]. However there are a number of omissions such as the beneficial process of trial and error, temporal and deep learning, and divergent vs convergent thinking in relation to the above. There is also the higher existential level of thinking. A further complexity arises with [25] theory of Multiple intelligences which implies the belief that there are to be a total of nine intelligences [53], where each person possesses a unique combination with one being the more primary or dominant variable The impact technology is increasingly having with people's ability to read [12] has an agonizing impact on [25] linguistic intelligence. Linguistic intelligence involves people with strong writing and speaking skills, memorization and reading [25]. Other types of the nine intelligences includes; Spatial, Bodily kinaesthetic, interpersonal, naturalist, music, linguistic, existential, logicalmathematical and Intrapersonal. Since fuzzy set theory was introduced by [82] in the 1960's, which suggested that uncertainty originating from human thinking can affect scientific problems. Since then, fuzzy logic has been successfully used in working with numerous practical applications. According to [65] "Fuzzy set theory is a research approach that can deal with problems relating to ambiguous, subjective and imprecise judgments, and it can quantify the linguistic facet of available data and preferences for individual or group decision-making"' [65].

Fuzzy set theory applied to psychology might be interpreted to suggest the cognitive processing is basically estimation rather than based upon thresholders, or reliable ground truth values. If enough people in a sample behave as if their strength of belief varies nearly continuously with the stimulus variable in the statement to be believed, then the given hypothesis would be supported and the psychological reality of fuzzy sets would be made more evident [34].

In the context of human intelligences, certain leaps across different logics and types of intelligences [25] can be intuitive, biased and not always with consistent or accurate entails 
resulting in sometimes best guess scenarios. However being able to draw upon multiple intelligences and logic processes in an intuitive way is advantageous in deducing entailments even with unfamiliar problems. This intuitive switch between the combination of previous rich experiences, different intelligences and logics results in human visualisations of entailments that often result in desired outcomes. This has a number of different underpinning axioms as a result of different methods for human learning. In addition to the above comes the further complexity that different logics (Proposition logic, First-order logic, Temporal logic, Probability theory, Fuzzy logic, etc.) or combinations of logic's that can be applied in order to determine what entails. Given the above overview of the complexity of human intelligence, it is highly unlikely that the capabilities of AI, is able to fully replicate the hyper complex human mind.

Deep learning, which is a process of AI, has made the most progress in solving complex problems consisting of recognizing speech from multiple speakers and identifying patterns in increasingly large data sets [64]. Subsequently, deep learning is already in some instances, substantially enhancing human capabilities [53]. Thus, with gaining knowledge instantly, this can have a profound effect on [25] multiple intelligence theory, where "logical-mathematic intelligence" is enhanced at a significantly faster pace than non-technological services. Thus, AI and other forms of technology are providing this instantly, and are of particularly benefit to scientists, mathematicians and philosophers who rely on this form of intelligence [25]. As such this context based problem solving could be considered as constituting narrow AI.

When intelligence is considered, it is important to discuss [21] second prominent theory of learning; behaviourism. Behaviourism can be defined as "changes in either the form or frequency of observable performance" [21]. The importance of behavioural learning implies the notion of learning from previous failures and the effects this has on the strengthening of future behaviour [73]. Conditioning occurs through interactions with the environment, thus behaviorists believe that the responses to environmental stimuli are accountable for future actions [26]. This importantly offers a perspective whereby the ground truth value may not act as a predetermined end point. The approach of behaviourism can be linked closely to reinforcement learning discussed later [75].

Consequently, the importance of learning through practice and encourages how it is imperative one must learn through the practice of skills before they can be performed accurately [39]. The usage of technology could be in fact diminishing bodily-kinetic intelligence. Face to face communication [17] can also diminish due to technology assisted interaction. This form of intelligence is prominent within athletes, dancers and surgeons, who are effective at body movement, hand-eye coordination and physical control [25]. Consequently, with technology constantly advancing, it provides the threat of the loss of "bodily-kinesthetic intelligence". This discussion can be echoed with the theory of naturalist intelligence [25], the idea of nurturing and exploring with the environment. It can be feared that soon, with advancements of technology, this form of intelligence is unlikely to be diminished.

It could be considered that as our reliance on technology increased then certain types of human intelligence may diminish, whilst AI is exponentially increasing. One such area is that of behavioral learning, where intelligence can be heavily influenced by the notion of human interaction [77]. There has been particular discussion within literature on the negatives affects technology is creating affecting face-to-face communication. It has been found [8] that there had been a prominent decrease in face-to-face interaction amongst the youth, due to these individuals growing up with the internet as part of their everyday life during education, communication and entertainment. The lack of human interaction and communication would subsequently affect [25] "interpersonal intelligence' dimension. Interpersonal intelligence can be defined as people with good interactions and communication skills, thus with a strong understanding of people and the emotions and motivations surrounding them. People with a lack of verbal communications and interactions face a significant threat of a core loss of intelligence.

A potential solution to the intelligence issue is through the use of cognitive architectures. Instead of aiming to create systems that are skilled in one aspect of human cognition in limited contexts, architectural research can be used to provide rich guidance across multiple tasks and domains [44]. Cognitive architectures differ from expert systems as they provide "counts of intelligent behavior at the systems level, rather than at the level of component methods designed for specialized tasks" [44].

The idea of cognitive architectures is not new. Most prolifically, [51] has argued for their existence in his "twenty questions paper' with his program for cognitive modelling.

Conversely deep learning and voice recognition, response and translate systems, within the area of verbal communications and interactions, AI powered systems may prove beneficial for both life and business improving impact of voice assistants such as Apple's Siri, Amazon's Alexa and Google's Assistant are intertwined with the notion of deep learning networks, recognizing requests and providing instant answers [32]. Voice assistants are constantly being used to bridge the information gap between the ability to read and type, thus benefiting dementia sufferers providing a present voice willing to answer questions repeatedly without losing patience [32]. Additionally, voice assistants are constantly enhancing translation [32]. Google has recently launched a new set of earbuds, providing people with voice assistance for real-time voice translation, allowing users to gain hands-free audio translations [32]. Thus, it can be highly discussed the enhancement technology creates with human capabilities through the use of knowledge, thus emulating forms of intelligence [64].

\section{ISSUES WITH BASELINE / GROUND TRUTH VALUE}

The importance of baseline conditions in scientific research is a prominent part of literature on research techniques. A baseline serves as an important reference point from which progress can be tracked and is an integral part of 
Monitoring and Evaluation (M \& E) frameworks [50]. It can be referred to at various points of the study process, from preliminary hypothesis testing to mid-point reviews. A baseline condition allows for comparisons to be made [29]. There are also number of stakeholders, including governments, citizens, the private sector, Non-Ggovernment Organizations (NGOs), Civil Society, international organizations, among others, which are now focused on increased performance of policies, programmes, and projects, which calls for enhancing baseline ground truth accuracy via results-Based Monitoring and Evaluation (RBME). Fundamental assumptions in experimental research are (a) the components and parameters of the conditions are known, and (b) those conditions are implemented with consistency and accuracy [3]. These assumptions apply to baseline conditions as well as intervention conditions. Within experimental research, it is widely regarded that baseline conditions are important for determining end points. A baseline serves as a reference point and is dependent on the following conditions: that "(a) the components and parameters of the conditions are known, and (b) those conditions are implemented with consistency and accuracy [31]. Within AI research, there are no consistent parameters. Studies in the field of AI (list some) test the power of AI systems not by their ability to be AI, but to achieve pre designed goals.

It is stated [7] there is an issue which is commonly faced when dealing with Web-based concepts: namely, vague ontologies. Within the realm of computer science, ontologies are defined as "the definition of domain concepts (extensions) and the relations between them" [7]. To say that an ontology is vague is when concepts are not clearly defined, i.e with vague language. For example, in the case of this paper, Basic, Moderate, Complex and Advanced AI could be described as vague ontologies, since they do not reveal the intricacies of component parts. Frege (1906) cited it [60] highlights that a "concept must have a sharp boundary" in order to avoid doubt [60].

A key question pertains to how error is minimized in machine learning algorithm. This is being done through the running of the algorithm as many times as is necessary in order to compare model prediction with the ground truth value with subsequent adjustments of parameters that result in smaller error, over time the result should be an increase in prediction accuracy. However, when there are a multitude of possibilities for the initial error, with varying degrees of impact, then simply adjusting parameters is unlikely to be sufficient. In the context of reinforcement learning with neural networks whereby the networks based agent discovers complete quantum-error-correction strategies, there is still a need for measurement outcomes [24]. They also suggest that the combinatorially large search space presents a substantial challenge when attempting to find Q-E-C strategies from scratch without human guidance [24]. The suggested solution is a tier stage learning reliant on human guidance [24]. The optimization of algorithms which characterises machine learning, through guessing and guessing again until close to ground truth value is insufficient in comparison to deep learning.
The programming towards a predictable ground truth value negates the possibility of an alternative end point. The reliance on software code and the numerous parameter adjustments makes the processing and final outcome relatively distorted particularly when applied within unacceptable error domains. This pertains to non-acceptable error margins between the prediction and the ground truth values which on a mass scale may mean a small percentage of unacceptable errors. In addition whilst there are a number of halo claims in relation to $\mathrm{AI}$, in the main there are still substantial hurdles for AI to overcome before it is to be wholly relied upon. The premise here is that the capability of AI does not sufficiently compare to the breadth and hyper complexity of human thinking but only mirrors and advances narrow types of human thinking.

Deep Learning could be considered as better than Machine Learning due to the needlessness of Feature Extraction. Machine Learning models use feature extraction to determine whether a given picture shows a car or not and must first have the features of a car (shape, size, windows, wheels etc.) must be programmed into the algorithm. It was found that having latent features extracted using DSAE proved useful for driving behaviour visualization [6]. However if it important here to highlight the previously mentioned issues with data. A key to Deep Learning models is that they increase their accuracy with the increasing amount of training data, whereas traditional machine learning models such as SVM and Naive Bayes classifier stop improving after a saturation point. In relation to algorithm consistency and convergence then it becomes important that a "Bayes network belief propagation algorithm converges on a probability distribution dictated by probability theory or proving that a theorem prover is sound and complete with respect to a semantics for some logic" [13].

In addition, recognition of the problems associated with neural networks being incredibly large and built by hand, has now been replaced with a technology which combines both human and AI to build the neural networks. As such this combination now accelerates the deep learning design for a number of applications including autonomous driving (Article - Researchers find a way to Harness AI Creativity-Dramatic performance boost to Deep Learning). By leveraging human ingenuity and experience with the meticulousness and speed of AI has a major contribution to on-the-edge deep learning solutions (Article - Researchers find a way to Harness AI Creativity-Dramatic performance boost to Deep Learning).

The application of deep learning methods have resulted in impressive advances in NLP, especially in the development of unsupervised models using recurrent neural networks and auto encoders that reduce dependence on high-quality, manual annotations of text data [18]. The methods of applying deep learning on electronic health records in Swedish to predict healthcare-associated infections [35] allows algorithms to learn high-level abstractions from clinical data and notes when concepts are not mentioned explicitly [66]. Availability of large volumes of real-world clinical data enables the training, development, and validation of new algorithms [71].

Beyond NLP, advances in machine learning have enabled new approaches for prediction of disease onset and future diseases [49]. This is in addition to the [81] exploration of 
machine learning techniques in predicting multiple sclerosis disease course. Another application is in image recognition for classification of radiology and pathology images [58]. Further applications include [28] development and validation of a deep learning algorithm for detection of diabetic retinopathy in retinal fundus photographs [22]. Dermatologist-level classification of skin cancer with deep neural networks, methods for assessing disease heterogeneity and predicting patient outcomes, given the information about a patient, their history, and individual-specific variability, have demonstrated capabilities to include both observed as well as latent features extracted from messy, multivariate EHR data [63]. Advanced analytics using machine learning on longitudinal RWD has the potential to inform and reframe drug development and clinical trial design strategy-through patient stratification into subgroups based on disease subtypes, drug treatment efficacy, progress, side effects, and toxicity profiles-by shifting from presumption of a single disease to multiple, related diseases. As machine-learning algorithms and frameworks continuously advance, there will be improvements in the ability of these models to learn continuously as new information emerges either in the form of additional data sources or updated treatment guidelines [19].

However there are issues in the application of NLP according to [71] where NLP methods is one approach to enable extraction and conversion of unstructured information from clinical text data to structured observations, to extraction of findings within ejection fraction from laboratory reports, biomarker information from pathology reports. In addition, the capture and use of patient characteristics such as emotional and social behaviors from physician notes [71]. Furthermore there is a central problem in that predefined fields in EHR (e.g., problem lists, past history, or test result fields) capture only certain disease information and may miss the trends of other prevalent, but unlisted, health conditions. NLP can be a powerful tool to extract symptoms from physician notes or textual data from lab reports to enable identification of those trends/conditions, thus complementing the assessments using structured data. The thoroughness and cross examination capabilities of a fully trained physician offers a multi-level health examination with a safety net consciousness such as AI safety [78] whereby a possible solution is where necessary verification with physicians can be sought. Thus beyond current AI capabilities.

The intelligence puzzle (as coined by [13] is an obstacle unique to the development of AI systems that seek to replicate "human-level artificial intelligence" (HLAI). In current science, we cannot say how "a system composed of unintelligent parts (such as neurons or transistors) can behave as intelligently as people" [13]. Thus it may be difficult to project this understanding of natural laws unto artificial laws and seek to recreate these systems in technology.

Furthermore, human intelligence is implicated in several fields: from economic policy to organisational practice and culture and society. As such, HLAI should be considered an atypical science [13]. Reinforcing this notion is that the goals of HLAI are more ambitious than the goals of its scientific counterparts. For example, medicine is often described as "the science or practice of the diagnosis, treatment, and prevention of disease" as opposed to "artificial immortality" (p.38). Resultantly, progress in achieving the latter seems minimal compared to the former: if we were to give AI (or HLAI in this case) a similarly defined goal, there would be fewer questions of progress made in the field [13].

It is not uncommon for terms that have entered the public domain from academia to be followed by clouds of misunderstanding and confusion [40]. In the case of HLAI (or AI in general), this bewilderment extends to the scientists involved in the field. Simply put: "the idea that our era is somehow seeing the emergence of intelligence in silicon that rivals our own entertains all of us, enthralling us and frightening us in equal measure. And, unfortunately, it distracts us", [40].

Scholars [80] argue that NLP is no longer valid or relevant, however. This is for the following reasons: 1. It is formed based on speculation "that the mind processes information at or below the Turing Limit', That it overlooks the (purported) reality that cognition surpasses computation. In its place, there should forms of "cognitive modelling untrammelled by standard computation" (p. 627) such as elements of hypercomputation like "analog chaotic neural nets, trial-and-error machines, Zeus machines" and so on. Supporting this notion are [10] and [67].

A slightly less critical view of NP comes from [74] who argue that while Newell's ideas about an amalgamated Theory of Mind (ToM) are not wrong, his methodology is flawed. Rather than altering existing approaches to meet criterion within NP, we should hold the complexities of the human mind in high regard, while developing "complementary theories at both psychological and connectionist levels, and cross-validate them" [74].

\section{CRoss Domain INTELLIGENCE}

An important area for consideration regarding a new approach to AI classification has to come from cross domain thinking. This includes the use and modelling of intelligence via examples from the natural world. There are a number of examples where significant breakthroughs regarding thinking and problem solving has been better achieved from things that exist in the natural world. For example there have been profound breakthroughs using Lobster (meridional) Eye Technology; for nanotechnology [56], Materials Science and in particular laser technology [46], Scientific Apparatus such as telescopes [16], [33], Physics [4]. In relation to the development of algorithms for predictive modelling, based on the 'collective intelligence' concept, contributions have come from Entomology and Ant Colony Optimisation [37] and Particle Swarm Optimisation, are the two most commonly known nature inspired algorithms. In addition the use of Bee's for Artificial bee colony-based predictive control for nonlinear systems [62]. An additional contribution comes from the context bio-inspired computation algorithm, in particular AI based optimization algorithm inspired from the nature of vortex [52]. It is the authors' belief that new classifications should cater for the various realms and combinations thereof, that has potential for beyond human intelligences. 


\section{KEY THEMES ON AI AND INTELLIGENCE}

There are a variety of issues pertaining to AI that includes not having any agreed standard categories from which to bind the different levels of AI. An additional set of issues is the lack of training and subsequent knowledge within the domain of human intelligence. Furthermore, there are issues with Data accuracy, Data issues with combinationally large search space. There are issues with quantum-error-correction strategies in addition to prediction errors against ground truth values in a variety of domains as presented herein. In particular the transparency of the level and type of error correction strategies should be critically considered particularly in critical domains such as healthcare. Early warning scoring [30] might enable contingencies to be enacted. The future direction and potential from cross domains has been discussed and remains a key area to consider in the AI classifications. In addition the authors recognise the level of human interaction in the design and management is also important. The above categorisations and definitions of AI offers a basis for informing a better standardisation of AI, and help to fulfil the purpose of this paper. Given the variability of the above the authors propose that there will be some level of confusion and lack of understanding from the data scientists' panel.

\section{RESEARCH PANEL AND SAMPLE}

Since this paper seeks to represent perceptions of AI, from the experts in data science, a non-random purposive sample was used. Data scientists/ experts were contacted to ensure theoretical, logical and analytical assumptions could be made, by applying expert knowledge to a cross-section of the population. Purposive sampling was used in order to ascertain perceptions from those working in the field of data science. The domain of data science being a thorough industry with its own unique screening process. By asking data scientists/ experts, the authors hope to gain an insight into how industry perceives, approaches, and classifies AI.

The authors have defined data scientists/ experts as those who are employed by corporations to work in machine learning, software development and traditional research, with responsibilities that can include data mining, algorithm development and/or data managing. By capturing the opinions of data scientists, modern perceptions of AI in the workplace can be gathered effectively. This is because, due to the comprehensive nature of data science, it is possible to gain insights into computer science, business knowledge and statistics respectively.

\section{RESEARCH FINDINGS}

We surveyed a group of experts (both male and female) aged 23-45, in professions such as data mapping, software engineering and data science. The majority of participants have been directly involved with AI for at least 2 years, with 4 being involved for 3+ years. When asked, "To what extent are you involved with AI?" on a scale of 1 (low involvement) to 5 (high involvement), participants had, on average, reported 3 (moderate involvement). When asked, "Do you consider yourself as having an advanced understanding of AI?", only 5 out of 20 answered with a "yes". Though this tended to correspond with the level of involvement, this was not always the case. For example: one participant who had been working with AI for 3+ years (with a high involvement of 4) answered this question with "to some degree", while another with high involvement (4) answered with "no" when asked about their understanding of AI.

Therefore, the authors conclude that length of time and extent of involvement do not necessarily relate with ideas about own understanding. While this uncertainty could be due to modesty or the general notion that "true knowledge is knowing you still have more to learn', it points to the wider issue of ambiguity behind the umbrella term "artificial intelligence", This supports the general claims made in this paper so far, and calls for a revision of terms. As a general observation, responses tended to become less specific throughout the various stages of the questionnaire, more specifically, as terms progressed through basic, moderate, advanced and complex AI.

\section{AI?}

Question 1: What do you consider as low level basic

When asked "what do you consider as low level basic AI?" responses ranged from "smartphones" to "supervised learning within a single-layer neural network"'. These variances indicate that even at a basic level, there is a lack of general consensus as to what constitutes AI. Only the minority of participants answered "Machine learning", "Automated learning", "Supervised learning via a neural network", akin to a combination of basics of AI and complex AI. Another example provided was "AI based on inputs and outputs" and "building a playable video game containing an AI opponent (The complexity of the game will correspond to the "level' of the AI"). The former suggests an understanding of grounding principles of machine learning while the latter indicates an idea of creativity being an important parameter. It also indicates that the individuals surveyed have low confidence and a low understanding of what constitutes AI. Another minority answered with Consumer products and services, such as Netflix, Facebook Messenger and Spotify Discover Weekly were all listed as being low level basic AI, as well as product features such as "red-eye reduction", and "camera lens focus". This consumer product answers were all given as a stand-alone halo answer without any real underpinning justifications. In addition to the above, "automation of tasks", was mentioned as low level basic AI, as well as "AI based on inputs and outputs". This notion ties into ideas about basic AI being operation-led (list scholars here) and designed with a clear goal in mind. The limitation can be extrapolated to be the inability to deviate from the goal, or to think independently. Hence, the element of independent thought will be carried forward to inform the overall design and level of AI.

More statistical functions such as "clustering" were also given, as well as "social media algorithms", and search/ return functions like "metadata tagging". This ties into the literature on AI that notes how "in their simplest form, intelligent agents are merely programs that solve specific problems". Already, it is visible that perceptions of what constitutes low level basic AI are, superficial in some cases, inaccurate and overall divided. 


\section{Question 2: What is moderate level AI?}

When asked about moderate level AI, learning emerged as a prominent theme in the form of "trained systems", "learning algorithms" and "supervised and unsupervised learning". In some cases, examples were given, for example, "regression, classification and decision trees" (within supervised and unsupervised learning) and "multi-layer neural networks" (within unsupervised learning specifically). Similar to the aforementioned, "structural equations" were also listed as being an example of moderate level AI, introducing modelling as a concept.

In one case, the concept of forecasting was noted, with the answer of "using AI to predict the stock market". This indicates that using imperfect (incomplete) information is a factor in an AI system's complexity, and is an element which will be carried forward to later sections. Similarly, another respondent answered with "boosted methods, ensemble methods" (meta-algorithms), indicating that bias reduction and combined ML techniques differentiate basic to moderate AI, additional factors to consider when making recommendations.

Specific examples of consumer products appeared throughout the answers, such as Google Home, Amazon Alexa and IBM's Watson. Similar to branded consumer products, functionalities such as "voice search" were also listed, as well as unbranded goods, like "smart homes". This follows on from the findings in Question 1, since it reinforces this notion that individuals tend to think of $\mathrm{AI}$ in terms of its benefits and personal gain. In one case, AlphaGo was listed as being an example of moderate AI (thus indicating a theoretical as well as commercial understanding of AI systems), though the former greatly surpassed the latter in volume. One participant even answered with "not sure as I'm unfamiliar with the different levels of AI", suggesting a need for universal definitions.

\section{Question 3: What do you consider as complex AI?}

When asked "What do you consider as complex AI?", three respondents listed "neural networks" as an example. In one respondent's answer, this was accompanied with a use case, specifically: "utilising unsupervised neural networks to provide simple value, i.e. recommendations". In another, "deep neural networks / unsupervised learning" were mentioned together. Unlike previous answers, "deep" appeared as a distinctive factor (at least in the respondent's mind) of what differentiated moderate AI and complex AI.

Once again, learning emerged as a prominent theme, but with a new dimension of "unassisted learning/ direction". This ties into the aforementioned operation on imperfect information, and will be considered in later sections. In a similar manner, one respondent listed "AI based on Artificial learnings and little to none input" as an example of complex AI. This supports classifications from (insert scholar from above). From this, themes of independent thought can be extrapolated, as a component of complex AI.

Following on from independent thought, an additional element of creativity can be extrapolated, as seen in the case of "coding/ creating/ consulting" and "creative machines".
This supports ideas about creativity being a feature in the classifications of more developed systems (Kaplan and Haenlein 2019). Beyond creativity, "voice emotion identification" was also introduced as a concept as well as "image" and "speech" recognition. This indicates that perceptions of complex AI systems entail the processing of unstructured data.

\section{Question 4: What do you consider as advanced AI?}

When asked about advanced AI, respondents tended to become less confident in their answers. For example, one participant responded with "robots?" and another with "something beyond that of a human mind". Several times, "I don't know" or "same as before" was listed as the answer, indicating uncertainty in own understanding, while the answers "creating AI" and "a fully conscious being - not there yet" indicate doubt in the field as a whole in terms of progress. However, unlike previous questions, one participant did answer with "General Intelligence", indicating an understanding of existing AI classifications. Other respondents did not refer to such classifications, indicating a lack of general consensus, even among experts in the field.

Once again, independent thought and creativity were raised as important attributes, with "AI based solely on its own learnings without input from humans" and "creative systems able to think independently". For another respondent, the similarity of AI systems compared to a human baseline was an indicator of an AI systems advancedness: "creating a machine to have a similar level of rounded intelligence like a human. Enabling it to think logically, learn and grow". Consciousness was also introduced as a concept, as well as "full autonomy": introducing the notion of active brain state achieved and self-directed goal pursuits. This human tendency to anthropomorphize could serve as an important measure of identifying (universally) what constitutes AI systems of varying degrees of complexities, thus will be carried forward.

\section{Overall: Questions Summary}

From doing the above analyses, it is apparent that there is little to no shared consensus of what constitutes varying degrees of AI classifications. Albeit, there are clear themes within each section, indicating some transference of thinking, though these are not guaranteed. This could be due to vague ontologies in the questionnaire design such as "low level" or "advanced", words which are subjective in nature - an error which the authors acknowledge. However, in search for a better word - this reinforces the need for a collective set of definitive parameters which can be used by individuals to form their ideas upon. There are potentially lexical gaps that need filling, or clear boundaries set from a respected source that help guide and inform the answers of individuals.

\section{CONCLUSIONS}

Conclusion - do the parameter adjustments need to be transparent re AI?

Conclusion - better training of data scientists to eliminate ground truth value differences and of professionals to be aware of the compounded adjustments is a recommendation of this paper. When considering the vast pace, various labels and 
variety of AI, combined with the complexity of the domain of human intelligence, the responses by the data scientists becomes partially explainable. In addition the domain of data science with the roles within it being generally narrow, offers another explanation of the paucity of response.

In addition the low confidence displayed by the data scientists should have been expected given the lack of training on the domain of human intelligence. The traditional background of data scientists is generally that of computer science. This is also in relation to the rate of change.

The current classification and diversity of terms and labels surrounding AI are too broad and overarching and may partially explain the lack of clarity and ease of which the public the attaches their worst case scenario's to the concept. The fog which surrounds AI is due in part to the hyper complexity that exists and the range of labels, categories and classifications that reduces transparency both in the research being conducted and the how it is communicated. Furthermore the use of a variety of realms from which to draw new, beyond human intelligences adds a further complication and challenge that needs to be addressed.

The concept of a sharp boundary coined by Frege [60] in 1906 applied to AI classification is further increased by the following, Hierarchy of AI. This the authors believe better captures the variety, complexity, interoperability and future of AI.

Subsequent use of the hierarchy of AI and the associated labels will provide greater transparency leading to improved understanding and the locating of research (both national and international) being undertaken within it.

\section{DIMENSIONS OF AI}

The following model (Fig. 1) demonstrates broadly the proportionality and scope of intelligences at each level. Overall it provides relatively sharp boundaries [60] between the levels. It caters for the latest developments and interoperability of AI in addition to the future potential. Thus acts as a basis for identifying potentially confusing areas and in some cases dangerous developments within AI, thus serving an agenda for understanding, regulation and transparency. It is important to note that the authors depict the hierarchy in the shape of an inverted pyramid to better capture the nature of AI. The following provides a level of explainability regarding the Hierarchy of AI. The first level is termed Systems intelligence (Table I shows the factors that constitute this level). The second level is termed Neural intelligence (Table I shows the factors that constitute this level). The top level considers the nature of multi-layered synthetic networks and interoperability thereof, whereby the systems and algorithms have the capability to exchange and make use of a variety of information types, across new boundaries, extends the level of risk, including risk censoring [20], and difficulty regarding the potential transparency of the error correction strategies. That is in addition to potential outcomes. As such the authors highlight this type of AI at the top of the AI Hierarchy. This type is termed Transversal intelligence. The hierarchy importantly depicts the level and combination of multiple error correction strategies due to the interoperability across data systems and data types. As such this Transversal level of intelligence is where it becomes imperative that there is a high level of regulation and transparency.

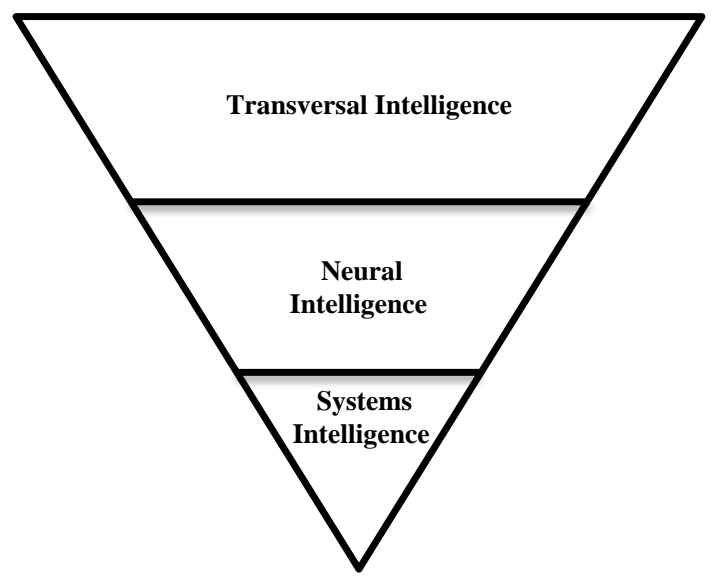

Fig. 1. Model: Hierarchy of AI.

TABLE I. EXPLANATIONS OF THE LEVELS AND CRITERIA WITHIN THE HIERARCHY OF AI

\begin{tabular}{|c|c|c|}
\hline \multicolumn{3}{|c|}{ Hierarchy of AI } \\
\hline Level & Overview & Key Parameters \\
\hline $\begin{array}{l}\text { Transversal } \\
\text { Intelligence }\end{array}$ & $\begin{array}{l}\text { Creative } \\
\text { Intelligence }\end{array}$ & $\begin{array}{l}\text { Typically no - minor human } \\
\text { involvement. Range of ability to } \\
\text { intelligently expand the data sets to new } \\
\text { domains automatically. Extreme level of } \\
\text { interoperability. Ability to determine } \\
\text { new; end points, rules, laws and beyond } \\
\text { human intelligence. }\end{array}$ \\
\hline $\begin{array}{l}\text { Neural } \\
\text { Intelligence }\end{array}$ & $\begin{array}{l}\text { Adaptive } \\
\text { Intelligence }\end{array}$ & $\begin{array}{l}\text { Typically no - Moderate human } \\
\text { involvement. Range of ability to } \\
\text { intelligently expand the data sets within } \\
\text { human set parameters and across human } \\
\text { domains. Machine and Deep Learning } \\
\text { interoperability across human levels of } \\
\text { data abstraction. Enabling Forecasting } \\
\text { and Predicting within the scope of } \\
\text { human intelligence. Combines Narrow, } \\
\text { deep and broad domains informing } \\
\text { results. }\end{array}$ \\
\hline $\begin{array}{l}\text { Systems } \\
\text { Intelligence }\end{array}$ & $\begin{array}{l}\text { Non adaptive } \\
\text { Systems } \\
\text { Intelligence }\end{array}$ & $\begin{array}{l}\text { Typically hi level of human } \\
\text { involvement. In ability of systems to } \\
\text { expand the data sets automatically. } \\
\text { Narrow and shallow in domains. }\end{array}$ \\
\hline
\end{tabular}

\section{REFERENCES}

[1] Acharya, P. and Mathur, M. (2020), Artificial intelligence in dermatology: the "unsupervised" learning. Br J Dermatol. Accepted Author Manuscript. doi:10.1111/bjd.18933

[2] Agre, P. (1997) "Toward a critical technical practice: lessons learned in trying to reform AI". In Beyond the Great Divide: social science, technical systems, and cooperative work, Edited by: Bowker, G. C., Gasser, L., Star, S. L. and Turner, W. 131-58. Mahwah, NJ: Erlbaum.

[3] Anderson, J.R., and Lebiere, C. (2003) The Newell Test for a Theory of Cognition, Behavioral and Brain Sciences, Vol.26, pp587-640

[4] Aslanyan V; Keresztes K; Feldman C; Pearson JF; Willingale R; Martindale A; Sembay S; Osborne JP; Sachdev SS; Bicknell CL; Houghton PR; Crawford T; Chornay D, The Review Of Scientific Instruments [Rev Sci Instrum], ISSN: 1089-7623, 2019 Dec 01; Vol. 90 (12), pp. 124502; Publisher: American Institute Of Physics; PMID: 31893794 
[5] Bart A., and Feigenbaum EA,. (2014), The handbook of artificial intelligence,

[6] Bichicchi, A. et al. (2020) 'Analysis of Road-User Interaction by Extraction of Driver Behavior Features Using Deep Learning', IEEE Access, Access, IEEE, 8, pp. 19638-19645. doi: 10.1109/ACCESS.2020.2965940.

[7] Bourahla M. (2015) Exact Reasoning over Imprecise Ontologies. In: Amine A., Bellatreche L., Elberrichi Z., Neuhold E., Wrembel R. (eds) Computer Science and Its Applications. CIIA 2015. IFIP Advances in Information and Communication Technology, vol 456. Springer, Cham

[8] Brignall and Van Valley (2005) the impact of internet communications on social interaction, Volume 25, 2005 - Issue 3,

[9] Bringsjord,S. and Schimanski B,. (2003) What is Artificial Intelligence, psychometric AI as an answer, Proc. of the 18th International Joint Conference on Artificial Intelligence (IJCAI 2003)

[10] Bringsjord, S. and Zenzen, M. (2003) Superminds: People harness hypercomputation, and more, Studies in Cognitive Systems, Springer, Vol.29,

[11] Caliskan, C, Bryson, J.j. Narayanan, A (2017) Semantics derived automatically from language corpora contain human-like biases, Science 14 Apr, Vol. 356, Issue 6334, pp. 183-186

[12] Carr, N., 2008. Is Google making us stupid? What the Internet is doing to our brains (Vol. 1). July

[13] Cassiamatis, N.L. (2012) "Artificial Intelligence and Cognitive Modelling Have the Same Problem", Theoretical Foundations of Artificial General Intelligence Atlantis Thinking Machines, vol. 4, pp. $11-24$,

[14] Cave, S., Dihal, K. (2019) Hopes and fears for intelligent machines in fiction and reality. Nat Mach Intell 1, 74-78

[15] Chollet, F. (2019) On the Measure of Intelligence, arXiv:1911.01547 [cs.AI]

[16] Daniel, V. et al (2019). In-Orbit Commissioning of Czech Nanosatellite VZLUSAT-1 for the QB50 Mission with a Demonstrator of a Miniaturised Lobster-Eye X-Ray Telescope and Radiation Shielding Composite Materials, Space Science Reviews. Aug 2019, Vol. 215 Issue 5, pN.PAG-N.PAG. 1p. DOI: 10.1007/s11214-019-0589-7,

[17] Drago, E., (2015). The effect of technology on face-to-face communication. Elon Journal of Undergraduate Research in Communications, 6 (1).

[18] Dubois, S., Romano, N. (2017) Learning effective embeddings from medical notes, arXiv preprint arXiv:1705.07025.

[19] Dorajoo, S.R. \& Chan, A. (2018) Implementing clinical prediction models: pushing the needle towards precision pharmacotherapy. Clin. Pharmacol. Ther. 103, 180- 183

[20] Dyagilev K, Saria S. (2015) Learning (predictive) risk scores in the presence of censoring due to interventions. Mach Learn 2016;102:32348.doi:10.1007/s10994-015-5527-7

[21] Ertmer, P.A. and Newby, T.J., (1993). Behaviorism, cognitivism, constructivism: Comparing critical features from an instructional design perspective. Performance improvement quarterly, 6(4), pp.50-72.

[22] Esteva, A., Kuprel, B., Novoa, R., Ko, J., Swetter, S., Blau, H., and Thrun, S. (2017). Dermatologist-level classification of skin cancer with deep neural networks. Nature, 542(7639):115-118.

[23] Fogel D.B. (1995) Evolutionary Computation Toward a New Philosophy of Machine Intelligence, 3rd Ed, Wiley and Sons

[24] Fosel, T., Tighineanu, P,. Weiss, T., and Marquardt, F. (2018), Reinforcement Learning with Neural Networks for Quantum Feedback, Physical Review X, Vol.8, Issue 3, July-Sept.

[25] Gardner, H., 1992. Multiple intelligences (Vol. 5, p. 56). Minnesota Center for Arts Education.

[26] Greer, D. R., Dudek-Singer, J. and Gautreaux, G. (2006), Observational learning. International Journal of Psychology, 41: 486-499. doi: $10.1080 / 00207590500492435$

[27] Gudwin, R.R. (2000) Evaluating intelligence: a computational semiotics perspective, Smc 2000 conference proceedings. 2000 ieee international conference on systems, man and cybernetics. 'cybernetics evolving to systems, humans, organizations, and their complex interactions'8-11 Oct 2000.

[28] Gulshan V, Peng L, Coram M, et al. (2016) Development and Validation of a Deep Learning Algorithm for Detection of Diabetic Retinopathy in Retinal Fundus Photographs. JAMA. 2016;316(22):2402-2410. doi:10.1001/jama.2016.17216

[29] Gusnard, D.A. and Raichle, M.E. (2001) Searching for a baseline: functional imaging and the resting human brain, Nature Reviews Neuroscience, Vol.10, pp685-694

[30] Henry, K. E, Hager, D. N, Pronovost, P. J, and Suchi, S. (2015) A targeted real-time early warning score (trewscore) for septic shock. Science Translational Medicine, 7(299 299ra122): 1-9,

[31] Horner, R. H., Carr, E. G., Halle, J., McGee, G., Odom, S., \& Wolery, M. (2005). The Use of Single-Subject Research to Identify EvidenceBased Practice in Special Education. Exceptional Children, 71(2), 165179.

[32] Hoy, M.B., 2018. Alexa, siri, cortana, and more: An introduction to voice assistants. Medical reference services quarterly, 37(1), pp.81-88.

[33] Inneman, et al, (2019) see Nentvich, O. j., \& Urban, M., \& Blažek, M., \& Inneman, A., \& Hudec, R., \& Sieger, L. (2019). Lobster eye optics: position determination based on 1D optics with simple code mask. 31 . 10.1117/12.2528505.

[34] Jackson, S., and Jacksina, C.M. (2018) Impact of Fuzzy Techniques in Psychology, International Journal of Recent Research Aspects, April 2018, pp491-494

[35] Jacobson, O., and Dalianis, H. (2016) "Applying deep learning on electronic health records in Swedish to predict healthcare-associated infections", Proc. 15th Workshop Biomed. Natural Lang. Process., pp. 191-195,

[36] Jajal, T. D. (2018) Distinguishing between Narrow Ai, General Ai, and Super $\mathrm{Ai}$, Medium, Artificial Intelligence. Accessed online https://medium.com/@tjajal/distinguishing-between-narrow-ai-generalai-and-super-ai-a4bc44172e22

[37] James, L. (2018) 5 Ways mother nature inspires artificial intelligence, Towards Data Science, Jan 14, 2018. Accessed 12/02/2020 https://towardsdatascience.com/5-ways-mother-nature-inspires-artificialintelligence-2c6700bb56b6

[38] Jiang, F., Jiang, Y., Zhi, H., Dong, Y., Li, H., Ma, S., Wang, Y., Dong, Q., Shen, H. and Wang, Y., (2017) Artificial intelligence in healthcare: past, present and future. Stroke and vascular neurology [online], 2 (4), 230-243.

[39] Johnson, R.T. and Johnson, D.W., 2008. Active learning: Cooperation in the classroom. The annual report of educational psychology in Japan, 47, pp.29-30.

[40] Jordan SC, et al. (2019) Daratumumab for Treatment of AntibodyMediated Rejection in a Kidney Transplant Recipient [abstract]. Am J Transplant. 2019; 19 (suppl 3).

[41] Kade, L., and Maltzan, S.V. (2019) Towards a Demystfication of the Black Box - Explainable Ai and the Legal Ramifications, Journal of Internet Law, September

[42] Kaplan A, Haenlein M. (2019) Siri, Siri, in my hand: Who's the fairest in the land? On the interpretations, illustrations, and implications of artificial intelligence. Business Horizons; 62:15.

[43] Krafft PM, Young M, Katell M, et al. (2019) Policy versus Practice: Conceptions of Artificial Intelligence. SSRN Electronic Journal. DOI: 10.2139/ssrn.3431304.

[44] Langley, P. (2019) Explainable, normative, and justified agency. In Proceedings of the Thirty-Third AAAI Conference on Artificial Intelligence, 2019.

[45] Legg, S. and Hutter, M. (2007) Universal Intelligence: A Definition of Machine Intelligence, Minds and Machines, Vol.17. Issue 4, pp391-444

[46] Lin, Kaijie; Yuan, Luhao; Gu, Dongdong. (2019) In Journal of Materials Processing Tech.. May 2019 267:34-43 Language: English. DOI: 10.1016/j.jmatprotec.2018.12.004, Database: ScienceDirect

[47] McCulloch, W.S. and Pitts, W. (1943) A logical calculus of the ideas immanent in nervous activity, The Bulletin of Mathmatical Biophysics, Vol.5, Issue 4, pp115-133 
[48] Miksad, R.A. \& Abernethy, A.P. (2018) Harnessing the power of realworld evidence (RWE): a checklist to ensure regulatory-grade data quality. Clin. Pharmacol. Ther. 103, pp.202-205.

[49] Miotto, R., Wang, F., Wang, S., Jiang, X., Dudley, J.T. (2016) Deep learning for healthcare: review, opportunities and challenges, Briefings in Bioinformatics, Volume 19, Issue 6, November 2018, Pages 12361246, https://doi.org/10.1093/bib/bbx044

[50] Moses, O. S. and Ssekamatte, D. (2016) Using baseline studies as a basis for monitoring and evaluation: A review of the literature, The Ugandan Journal of Management and Public Policy Studies Volume 11 Number 1 November 2016.

[51] Newell, A. (1973) You can't play 20 questions with Nature and win: projective comments on the papers of this Symposium, May 1973

[52] Onet, E.V. and Vladu, E., 2008. Nature inspired algorithms and Artificial Intelligence. Journal of Computer Science and Control Systems, (1), p.66.

[53] O’Neill, C. (2017) “The Ivory Tower Can’t Keep Ignoring Tech.” The New York Times, $17-$ Nov

[54] Oswald, E., 2019. What is artificial intelligence? Here's everything you need to know [online]. Digital Trends. Available from: https://www.digitaltrends.com/cool-tech/what-is-artificial-intelligenceai/ (Accepted 9 March 2019)

[55] Pedersen, T. \& Johansen, C.,( 2019) Behavioural Artificial Intelligence: An agenda for systematic empirical studies of Artificial Inference, Ai and Society, Springer London, pp1-14 https://doi.org/10.1007/s00146019-00928-5

[56] Peng, J.S., and Cheng, Q.F. (2017) High-Performance Nanocomposites Inspired by Nature, Advanced Materials, Vol.29, pp1-16

[57] Prasad, P., Pathak, R., Gunjan, V., \& Ramana Rao, H.V. (2019). Deep Learning Based Representation for Face Recognition. 10.1007/978-98113-8715-9_50.

[58] Ramagopalan, S.V et al (2017) Risk of Thrombosis in Sjögren Syndrome: The Open Question of Endothelial Function Immunemediated Dysregulation, Luca Quartuccio, The Journal of Rheumatology Aug 2017, 44 (8) 1106-1108; DOI: 10.3899/jrheum. 170462

[59] Razavian, A.S., Sullivan, J., Maki, A., and Carlsson.S. (2016) Visual instance retrieval with deep convolutional networks. CoRR, abs/1412.6574,

[60] Ricketts, T. (1997) Frege's 1906 Foray into Metalogic, Philosphical Topics, Vol.25. Issue 2, Fall, pp169-188

[61] Russell S.J. and Norvig P. (2016) Artificial Intelligence : A Modern Approach, Pearson Education Limited, London

[62] Sahed, O. A., Kamel, K., and, Abousoufyane. B. (2015) Transactions of the Institute of Measurement \& Control. Ju12015, Vol. 37 Issue 6, p780792. 13p. DOI: $10.1177 / 0142331214546796$

[63] Schulam, P., Wigley, F., and Saria, Suchi, S. (2015). Clustering longitudinal clinical marker trajectories from electronic health data: Applications to phenotyping and endotype discovery. In Proceedings of the Twenty-Ninth AAAI Conference on Artificial Intelligence,

[64] Sejnowski (2019) http://theconversation.com/artificial-intelligence-willmake-you-smarter-101296
[65] Shan, M., Chan, A. P., Le, Y., \& Hu, Y. (2015). Investigating the effectiveness of response strategies forvulnerabilities to corruption in the Chinese public construction sector. Science and EngineeringEthics, 21(3), 683-705.

[66] Shickel, B., Tighe, P. J., Bihorac, A. \& Rashidi, P. (2017) Deep EHR: a survey of recent advances in deep learning techniques for electronic health record (EHR) analysis. IEEE J. Biomed. Health Inform. 22, 1589-1604.

[67] Siegelmann, H.T. (1999) Neural Networks and Analog Computation. Beyond the Turing Limit, Springer, Switzerland.

[68] Sinz, F., \& Bethge, M. (2008). The conjoint effect of divisive normalization and orientation selectivity on redundancy reduction in natural images. In Frontiers in Computational Neuroscience. Conference Abstract: Bernstein Symposium. doi:10.3389/conf.neuro.10.2008.01.116.

[69] Snelbecker, G. E. (1983). Learning theory, instructional theory, and psychoeducational design. New York: McGraw-Hill.

[70] Sweeney, L. (2003). That's AI?: a history and critique of the field. Technical Report, CMU-CS-03-106. Pittsburgh: Carnegie Mellon University, School of Computer Science

[71] Swift, B., Jain, L., White, C., Chandrasekaran, V., Bhandari, A., Hughes, D. A., et al. (2018). Innovation at the Intersection of Clinical Trials and Real-World Data Science to Advance Patient Care. Clin. Transl. Sci. 11, 450-460. doi: 10.1111/cts.12559

[72] SyncedReview (2018) 2018 in Review: 10 AI Failures https://medium.com/syncedreview/2018-in-review-10-ai-failuresc18faadf5983

[73] Walker, S., 2017. Learning theory and behaviour modification. Routledge.

[74] Wang. Y., and Chiew, V. (2010) On the cognitive process of human problem solving, Cognitive Systems Research, Vol.11, Issue 1, Mar, Pages 81-92

[75] Weegar, M.A. and Pacis, D., 2012. A Comparison of two theories of learning-behaviorism and constructivism as applied to face-to-face and online learning. In Proceedings e-leader conference, Manila.

[76] White, R. (2017) Building trust in real-world evidence and comparative effectiveness research: the need for transparency, J. Comp. Eff. Res. 6, $5-7$

[77] Wright, L. M., \& Leahey, M. (2000). Nurses and families: A guide to family assessment and intervention (3rd ed.. Philadelphia: F. A. Davis

[78] Yampolskiy, R.V. (2018), Artificial Intelligence safety and security, Taylor Francis, London

[79] Yampolskiy, R. V. (2019), "Predicting future AI failures from historic examples", Foresight, Vol. 21 No. 1, pp. 138-152.

[80] Yang, Y., and Bringsnord, S. (forthcoming) Mental metalogic: A new, unifying theory of human and machine reasoning. Erlbaum. Cited in Anderson, J.R., and Lebiere, C. (2003) The Newell Test for a Theory of Cognition, Behavioral and Brain Sciences, Vol.26, pp587-640

[81] Zhao, Y. et al (2017)Exploration of machine learning techniques in predicting multiple sclerosis disease course, PLoS ONE, 12, p. $\mathrm{e} 0174866$

[82] Zadeh L.A. (1965), Fuzzy Sets, Information and Control, 8, 338-353, 1965. 